\title{
Non-coordinate case of graded differential algebra with ternary differential
}

\author{
Nadezda BAZUNOVA \\ Department of Mathematics, Tallinn University of Technology \\ Ehitajate tee 5, 19086 Tallinn, Estonia \\ E-mail: nadezhda@staff.ttu.ee
}

This article is part of the Proceedings of the Baltic-Nordic Workshop, Algebra, Geometry and Mathematical Physics which was held in Tallinn, Estonia, during October 2005.

\begin{abstract}
In this article, we generalize a construction of graded $q$-differential algebra with ternary differential satisfying the property $d^{3}=0$ and $q$-Leibniz rule on the non-coordinate case, that is on the case where the differentials of generators of underlying algebra do not coincide with generators of bimodule of first order forms. Our starting point is the coordinate first order differential calculus on associative complex algebra $\mathcal{A}$ with $n$ generators and the bimodule of second order differentials.
\end{abstract}

\section{Introduction}

In this work, we investigate a generalization of coordinate graded differential algebra with ternary differential on an arbitrary complex associative unital algebra. Here we use the word "non-coordinate" following to terminology in [2] where "non-coordinate" means that the differentials of generators of algebra do not coincide with the generators of bimodule of first order forms. The construction of coordinate graded differential algebra with ternary differential on an arbitrary complex associative unital algebra was established in [1].

As in [1], our inspiration is twofold. On one hand, we have the approach of S. Woronowicz [3] in order to construct the quantum de Rham complex for an ordinary differential $\left(d^{2}=0\right)$. On the other hand, we generalize the method applied in $[4,5]$ for the universal differential calculus and $N$-ary differential, that is we use the idea that the condition $d^{2} \neq 0$ implies the appearing of higher order differentials. Starting from some first order (non-coordinate) differential calculus with right partial derivatives (see [2]), we extend this non-coordinate first order differential calculus by differential $d$ satisfying the property $d^{3}=0$ and the $q$-Leibniz rule:

$$
d(\omega \theta)=(d \omega) \theta+q^{n} \omega(d \theta),
$$

where $\omega \in \Omega^{(n)}, \theta \in \Omega$ and the complex number $q$ is assumed to be primitive third degree root of the unity, that is in our case $q=e^{i \frac{2 \pi}{3}}$ and $[3]_{q} \doteq 1+q+q^{2}=0$. But in our case, in order to construct graded differential algebra with ternary differential, we need in 
additional assumption on the second order forms: we assume that the second order forms generate a bimodule with free right structure.

Specifically for the case $d^{3}=0$, the generalization of classical differential calculus has been worked out and studied in [6], [7]. The differential calculus with differential $d^{3}=0$ on associative algebra with $n$ generators and quadratic relations, and on quantum plane specifically was constructed in [8]. Also such construction was realized on superspaces with one and two parametric quantum groups as symmetry groups in [9]. In the recently appeared paper [10], authors examine the construction of $N$-differential graded algebra with the usual Leibniz rule and moduli space of deformations of differential of $N$-differential graded algebras.

\section{Non-coordinate first order differential calculus with right partial derivatives}

In details, the non-coordinate first order differential calculus have been constructed and studied in [2]. Because we start from this non-coordinate first order differential calculus, now we briefly remind its construction.

Let $\mathcal{A}$ be an algebra, $\mathcal{M}$ be $\mathcal{A}$-bimodule, $d^{1}: \mathcal{A} \rightarrow \mathcal{M}$ be a linear mapping such that the Leibniz rule

$$
d^{1}(u v)=d^{1}(u) v+u d^{1}(v), \forall u, v \in \mathcal{A}
$$

is fulfilled, that is the linear mapping $d^{1}$ be a differential.

Definition 1. The triple $\left\{\mathcal{M}, \mathcal{A}, d^{1}\right\}$ is called a first order differential calculus (FODC) on an algebra $\mathcal{A}$ with values in $\mathcal{M}$. The elements of $\mathcal{M}$ are called one-forms.

In our case, the algebra $\mathcal{A}$ is a complex associative unital algebra generated by elements $\left\{x^{1}, x^{2}, \ldots, x^{n}\right\} \subset \mathcal{A}$; the bimodule $\mathcal{M} \doteq \mathcal{M}^{(1)}$ is a free right module with bases $\left\{\mu_{1}^{1}, \mu_{1}^{2}, \ldots, \mu_{1}^{n}\right\} \subset \mathcal{M}^{(1)}, n \in \mathbb{N}$; then the first order differential of any element $u \in \mathcal{A}$ can be uniquely defined by the following decomposition:

$$
d^{1}(u)=\mu_{1}^{k} D_{k}(u),
$$

where the linear maps $D_{k}: \mathcal{A} \rightarrow \mathcal{A}$ are called right partial derivatives. Clearly, that $D_{k}(1)=0$.

Denote $D_{k}\left(x^{i}\right) \doteq \sigma_{k}^{i}$. Since we are dealing with non-coordinate differential, we assume that $\sigma_{k}^{i} \neq \delta_{k}^{i}$ as distinction from coordinate case where $\sigma_{k}^{i}=\delta_{k}^{i}$ (as usual, $\delta_{k}^{i}$ is Kronecker delta). In our notation, we write the first order differential of generators of the algebra $\mathcal{A}$ as follows:

$$
d^{1}\left(x^{i}\right)=\mu_{1}^{k} \sigma_{k}^{i}, \quad i=1, \ldots, n .
$$

Let a structure of bimodule on the free right module $\mathcal{M}^{(1)}$ can be defined by means of the algebra homomorphism $\xi=\left(\xi_{k}^{j}\right): \mathcal{A} \rightarrow \operatorname{Mat}_{n} \mathcal{A}$ such that

$$
u \mu_{1}^{j}=\mu_{1}^{k} \xi(u)_{k}^{j}, \quad \forall u \in \mathcal{A}, j, k=1, \ldots, n,
$$


and then specifically on the generators of the algebra $\mathcal{A}$ :

$$
x^{i} \mu_{1}^{j}=\mu_{1}^{k} \xi\left(x^{i}\right)_{k}^{j}, \quad i=1, \ldots, n .
$$

It follows immediately from the Leibniz rule (2.1) that the partial derivatives $D_{k}$ and homomorphism $\xi$ are connected by formula:

$$
D_{k}(u v)=D_{k}(u) v+\xi(u)_{k}^{j} D_{j}(v), \quad \forall u, v \in \mathcal{A} .
$$

Further on, we assume that $\mathcal{M}^{(1)}=\left(d^{1} \mathcal{A}\right) \mathcal{A}$. This means that the $\mathcal{A}$-bimodule $\mathcal{M}^{(1)}$ is generated by the first order differentials (one-forms). Also we would like to emphasize that the property $\left(d^{1} \mathcal{A}\right) \mathcal{A}=\mathcal{A}\left(d^{1} \mathcal{A}\right)$ follows immediately from the Leibniz rule $(2.1)$.

\section{$3 \quad$ Graded differential algebra with $d^{3}=0$}

Firstly, let us give the definition of graded $q$-differential algebra with ternary differential $d^{3}=0$. Our definition agrees with the more general one proposed in $[4,5]$, where the graded universal $q$-differential algebra with differential $d^{N}=0$ was constructed.

Definition 2. The pair $\{\Omega, d\}$, where $\Omega=\oplus_{n \geq 0} \Omega^{(n)}$ is a graded algebra over the algebra $\mathcal{A}=\Omega^{0}$, and $d: \Omega \rightarrow \Omega, d=\oplus_{n \geq 1} d^{n}$ is a homogeneous linear map of grade one, where $d^{n+1}: \Omega^{(n)} \rightarrow \Omega^{(n+1)}$, such that the $q$-Leibniz rule

$$
d(\omega \theta)=(d \omega) \theta+q^{n} \omega(d \theta), \text { for } \omega \in \Omega^{(n)}, \theta \in \Omega,
$$

and the property $d^{3}=0$ are satisfied, is called a graded differential algebra with ternary differential. The elements of $\Omega^{(n)}$ are referred to as $n$-forms.

Before construction of such algebra for the case of non-coordinate calculus with right partial derivatives, since now we assume that $d \mu_{1}^{i} \neq 0$, we need to introduce elements $\mu_{2}^{i}$ of grade $2, i=1, \ldots, n \in \mathbb{N}$, such that $d \mu_{1}^{i}=\mu_{2}^{i}$ and $d \mu_{2}^{i}=0$. We will refer to these elements $\mu_{2}^{i}$ as second order differentials. Moreover, we also assume that elements $\left\{\mu_{2}^{1}, \mu_{2}^{2}, \ldots, \mu_{2}^{n}\right\}$ generate a bimodule $\mathcal{M}^{(2)}$ over $\mathcal{A}$.

Following the general idea of [2], we define a graded $\mathcal{A}$-bimodule $\mathcal{E}=\mathcal{M}^{(1)} \oplus \mathcal{M}^{(2)}$, and construct a tensor algebra of $\mathcal{A}$-bimodule $\mathcal{E}$ over algebra $\mathcal{A}$ :

$$
T_{\mathcal{A}} \mathcal{E}=\mathcal{A} \oplus \mathcal{E} \oplus \mathcal{E}^{\otimes 2} \oplus \mathcal{E}^{\otimes 3} \oplus \cdots=\bigoplus_{s \geq 0} \mathcal{E}^{\otimes s}
$$

where $\mathcal{E}^{\otimes s}$ means $\mathcal{E} \otimes_{\mathcal{A}} \cdots \otimes_{\mathcal{A}} \mathcal{E}$.

After expanding and rearranging terms in $T_{\mathcal{A}} \mathcal{E}$, we obtain an $\mathbb{N}$-graded algebra

$$
T_{\mathcal{A}} \mathcal{E}=\bigoplus_{s \geq 0} T^{s}
$$

with an unique graduation compatible with graduation of $\mathcal{E}$ (see [5]). Here $T^{0}=\mathcal{A}, T^{1}=$ $\mathcal{M}^{(1)}, T^{2}=\mathcal{M}^{(2)} \oplus \mathcal{M}^{(1)} \otimes \mathcal{M}^{(1)}$, and for $s>2$

$$
T^{s}=\bigoplus_{\substack{m \leq s, a_{i}=1,2 ; \\ a_{1}+\cdots+a_{m}=s}} \mathcal{M}^{\left(a_{1}\right)} \otimes_{\mathcal{A}} \cdots \otimes_{\mathcal{A}} \mathcal{M}^{\left(a_{m}\right)}
$$


consists all possible tensor products of the total grade equal to $s$.

Let us define a commutation rule between the elements of algebra $\mathcal{A}$ and bimodule $T^{2}$ with free right structure as it follows:

$$
u \mu_{2}^{j}=\mu_{2}^{k} \xi(u)_{k}^{j}+\mu_{1}^{k} \otimes \mu_{1}^{l}\left(q D_{l}\left(\xi(u)_{k}^{j}\right)-\xi\left(D_{k}(u)\right)_{l}^{j}\right) \quad \forall u \in \mathcal{A}, j, k=1, \ldots, n,
$$

and in particular for the generators of $\mathcal{A}$ :

$$
x^{i} \mu_{2}^{j}=\mu_{2}^{k} \xi_{k}^{i j}+\mu_{1}^{k} \otimes \mu_{1}^{l}\left(q D_{l}\left(\xi_{k}^{i j}\right)-\xi\left(\sigma_{k}^{i}\right)_{l}^{j}\right) \quad \forall u \in \mathcal{A}, j, k=1, \ldots, n,
$$

where $\xi_{k}^{i j} \doteq \xi\left(x^{i}\right)_{k}^{j} \in \mathcal{A}$. This means that from now on, we make the assumption: the bimodule $T^{s}$ is generated by the elements $\left\{\mu_{2}^{i}, \mu_{1}^{j} \otimes \mu_{1}^{k} ; i, j, k=1, \ldots, n\right\}$ and equipped with the structure of right $\mathcal{A}$-module.

Then, due to the fact that the tensor product in (3.3) is taken over the algebra $\mathcal{A}$, any homogenous element $v$ of grade $s$ has unique decomposition in the forms

$$
v=\mu_{a_{1}}^{i_{1}} \otimes \cdots \otimes \mu_{a_{m}}^{i_{m}} v_{i_{1} \ldots i_{m}},
$$

where $m \leq s, ; v_{i_{1} \ldots i_{m}} \in \mathcal{A}$ and the sum in (3.4) is over all possible choices of $a_{1}, \ldots a_{m}$, $a_{k}=1,2$, such that $a_{1}+\cdots+a_{m}=s$. We refer to such composition as canonical decomposition.

Now we are in position to define a grade one linear mapping $d: T_{\mathcal{A}} \mathcal{E} \rightarrow T_{\mathcal{A}} \mathcal{E}$ by:

$$
\begin{aligned}
& d\left(\mu_{a_{1}}^{i_{1}} \otimes \cdots \otimes \mu_{a_{m}}^{i_{m}} v_{i_{1} \ldots i_{m}}\right)= \\
& \sum_{j=1}^{m} q^{\left(a_{1}+\cdots+a_{j-1}\right)} \mu_{a_{1}}^{i_{1}} \otimes \cdots \otimes \mu_{a_{j-1}}^{i_{j-1}} \otimes \mu_{a_{j}+1}^{i_{j}} \otimes \mu_{a_{j+1}}^{i_{j+1}} \cdots \otimes \mu_{a_{m}}^{i_{m}} v_{i_{1} \ldots i_{m}}+ \\
& q^{\left(a_{1}+\cdots+a_{m}\right)} \mu_{a_{1}}^{i_{1}} \otimes \cdots \otimes \mu_{a_{m}}^{i_{m}} \otimes \mu_{1}^{j} D_{j}\left(v_{i_{1} \ldots i_{m}}\right) .
\end{aligned}
$$

But the simple calculation shows that

$$
\begin{aligned}
& d^{3}(u)=d^{2}\left(\mu_{1}^{j} D_{j}(u)\right)=d\left(\mu_{2}^{j} D_{j}(u)+q \mu_{1}^{j} \otimes \mu_{1}^{k} D_{k}\left(D_{j}(u)\right)\right)= \\
& q[2]_{q} \mu_{2}^{j} \otimes \mu_{1}^{k} D_{k}\left(D_{j}(u)\right)+q^{2} \mu_{1}^{j} \otimes \mu_{2}^{k} D_{k}\left(D_{j}(u)\right)+\mu_{1}^{j} \otimes \mu_{1}^{k} \otimes \mu_{1}^{l} D_{l}\left(D_{k}\left(D_{j}(u)\right)\right)
\end{aligned}
$$

does not vanish in general.

In addition, it should be noticed that the Leibniz rule (3.1) does not hold in general for the elements which are not written in the canonical form (3.4), although the definition (3.5) agrees with this Leibniz rule.

Because of these, the tensor algebra $T_{\mathcal{A}} \mathcal{E}$ equipped with the operator $d$, as defined above, is not a ternary $q$-differential algebra. Our aim now is to construct the minimal homogeneous ideal $I_{q}$ in $T_{\mathcal{A}} \mathcal{E}$ such that $d I_{q} \subset I_{q}$, and that the corresponding quotient algebra

$$
\Omega(\mathcal{A}, \mathcal{M}) \doteq \frac{T_{\mathcal{A}} \mathcal{E}}{I_{q}}=\bigoplus_{s \geq 0} \Omega^{(s)}(\mathcal{A}, \mathcal{M})
$$

becomes an $\mathbb{N}$-graded $q$-differential ternary algebra, i.e. one recovers $d^{3}=0$ as well as the $q$-Leibniz rule (3.1).

To this end, we shall follow the general technique developed in [2].

The basic relations are

$$
x^{i} \mu_{1}^{j}-\mu_{1}^{k} \xi_{k}^{i j}=0 \text { and } d x^{i}=\mu_{1}^{k} \sigma_{k}^{i} .
$$


Consecutive differentiation of (3.7) by means of (3.1) leads to the following two homogeneous elements in $T_{\mathcal{A}} \mathcal{E}$ :

$$
\begin{gathered}
x^{i} \mu_{2}^{j}-\mu_{2}^{k} \xi_{k}^{i j}-\mu_{1}^{k} \otimes \mu_{1}^{l}\left(q D_{l}\left(\xi_{k}^{i j}\right)-\xi\left(\sigma_{k}^{i}\right)_{l}^{j}\right), \\
\mu_{2}^{k} \otimes \mu_{1}^{l} D_{l}\left(\sigma_{k}^{i}\right)-q^{2} \mu_{1}^{k} \otimes \mu_{2}^{l} D_{l}\left(\sigma_{k}^{i}\right)-\mu_{1}^{k} \otimes \mu_{1}^{l} \otimes \mu_{1}^{m} D_{m} D_{l}\left(\sigma_{k}^{i}\right),
\end{gathered}
$$

which, in fact, are generators for $I_{q}$. Moreover, consecutive differentiation of (3.8) and (3.9) leads us to the additional conditions on the partial derivatives $D_{l}$ and homomorphism $\xi$ :

$$
D_{l} \circ D_{k}=D_{l} \circ \xi+\xi \circ D_{k} \quad \text { and } \quad \xi \circ D_{l} \circ D_{k}=0 .
$$

For example, the bimodule $\Omega^{(2)}(\mathcal{A}, \mathcal{M})$ has structure

$$
\Omega^{(2)}(\mathcal{A}, \mathcal{M})=\frac{\mathcal{M} \otimes \mathcal{M} \oplus \mathcal{M}^{(2)}}{I_{q}^{(2)}}
$$

where $I_{q}^{(2)}$ denotes a sub-bimodule in $T^{2}$ generated by relations (3.8). Now the differential $d^{2}: \mathcal{A} \rightarrow \Omega^{(2)}(\mathcal{A}, \mathcal{M})$ satisfies the q-Leibniz rule

$$
d^{2}(u v)=d^{2}(u) v+[2]_{q} d u d v+u d^{2} v, \quad \forall u, v \in \mathcal{A},
$$

as expected in [4].

We are thus led to the following

Theorem 1. Let $\Omega(\mathcal{A}, \mathcal{M})$ be the quotient algebra (3.6), where the homogeneous ideal $I_{q} \subset T_{\mathcal{A}} \mathcal{E}$ be generated by set of elements (3.7)-(3.10), and the operator $d: \Omega(\mathcal{A}, \mathcal{M}) \rightarrow$ $\Omega(\mathcal{A}, \mathcal{M})$ be defined by formula (3.5).

Then the pair $\{\Omega(\mathcal{A}, \mathcal{M}), d\}$ is a graded differential algebra with ternary differential.

Here we only give the main ideas of the proof. All details and proof will be presented elsewhere.

In order to prove Theorem 1, it is sufficient to show by induction on degree of elements in $T_{\mathcal{A}} \mathcal{E}$ that the following properties:

1. $d\left(I_{q}\right) \subset I_{q}$;

2. the $q$-Leibniz rule

$$
d(\omega \theta)=(d \omega) \theta+q^{n} \omega(d \theta) \quad\left(\bmod I_{q}\right)
$$

is satisfied $\forall \omega \in T^{n}$ and $\theta \in T_{\mathcal{A}} \mathcal{E}$;

3. $\left.d^{3}\left(T_{\mathcal{A}} \mathcal{E}\right)\right) \subseteq I_{q}$

hold in the tensor algebra $T_{\mathcal{A}} \mathcal{E}$. 


\section{References}

[1] Bazunova N, Construction of graded differential algebra with ternary differential, Cont. Math. 391 (2005), 1-11; math-ph/0509015.

[2] Borowiec A, Kharchenko V K, Algebraic approach to calculuses with partial derivatives, Sib. Adv. Math. 5 (1995), 10-37; q-alg/9501018.

[3] Woronowicz S L, Differential calculus on compact matrix pseudogroups (quantum groups), Commun. Math. Phys. 122 (1989), 125-170.

[4] Dubois-Violette M, Kerner R, Universal $Z_{N}$-graded differential calculus, J. Geom. Phys. 23 (1997), 235-246.

[5] Dubois-Violette M, Kerner R, Universal $q$-differential calculus and $q$-analog of homological algebra, Acta. Math. Univ. Comenianae LXV 2 (1996), 175-188; q-alg/9608026.

[6] Kerner R, $Z_{3}$-graded exterior differential calculus and gauge theories of higher order, Lett. Math. Phys. 36 (1996), 441-454; math-ph/0004032.

[7] Abramov V, Kerner R, Exterior differentials of higher order and their covariant generalization J. Math. Phys. 41 (2000), 5598-5614; math.QA/0004162.

[8] Bazunova N, Borowiec A, Kerner R, Quantum de Rham complex with $d^{3}=0$ differential, Czech. J. Phys. 51 (2001), 1266-1271; math-ph/0110007.

[9] Baz M El, Hassouni A El, Hassouni Y, Zakkari E H, $d^{3}=0, d^{2}=0$ differential calculi on certain noncommutative (super) spaces.J. Math. Phys. 45 (2004), 2314-2322; math-ph/0303057.

[10] Angel M, Díaz R, N-differential graded algebras, math.DG/0504398. 